Supplement of Biogeosciences, 12, 7057-7070, 2015

http://www.biogeosciences.net/12/7057/2015/

doi:10.5194/bg-12-7057-2015-supplement

(C) Author(s) 2015. CC Attribution 3.0 License.

(c) (i)

Supplement of

\title{
Carbon dynamics in boreal peatlands of the Yenisey region, western Siberia
}

E. D. Schulze et al.

Correspondence to: E. D. Schulze (dschulze@bgc-jena.mpg.de)

The copyright of individual parts of the supplement might differ from the CC-BY 3.0 licence. 


\section{Supplement}

\section{Supplement S1: Peat types and Bog surface}

Raised bogs have a slightly convex surface which can be covered by Pine-dwarf shrubsSphagnum vegetation ('ryam' bog) (bogs) with homogenous hummocky surface or extensive mire complexes with ridge-hollow patterns. On the gentle slopes (gradient $0.003-0.008 \mathrm{~m}$ $\mathrm{km}^{-1}$ ) vast ridge-hollow complexes exist, on the central flat part of large mire systems numerous shallow pools and small lakes develop. The vegetation comprises typical raised bog plant communities, where stunted (0.5 - $4 \mathrm{~m}$ high) pines (Pinus sylvestris) form more or less close tree layer or the trees are sparsely distributed on hummocks and ridges. (A subtype of the raised bog type is the "ryam" bog, which is specific and unique for Siberia. It is characterized by a tree layer of Pinus and dwarf shrubs with a Sphagnum and Monocot ground layer). Ericaceous dwarf shrubs such as Ledum palustre, Chamaedaphne calyculata, Andromeda polifolia on ridges and in 'ryams' are abundant. Eriophorum vaginatum and Carex limosa, as well as a few other species (Scheuchzeria palustris, Rhynchospora alba, Oxycoccus ssp., Drosera spp.) occur in waterlogged hollows. The ground layer is dominated by Sphagnum species, distributed following a moisture gradient with Sphagnum fuscum admixed with S. angustifolium, S. magellanicum on hummocks and S. balticum, S. jensenii, $S$. majus, S. lindbergi, S. papillosum in hollows (Lapshina and Vasiliev, 2001).

On sandy soils low stature Pinus sylvestris trees, dwarf shrubs and Eriophorum vaginatum are associated with the Sphagnum angustifolium vegetation establishes (with an admixture of $S$. magellanicum and $S$. fuscum). These communities presently form shallow Sphagnum peat deposits $(0.5-1.5 \mathrm{~m})$. Sometimes with a thin basal layer $(0.1-0.2 \mathrm{~m})$ of decomposed dense wood-cotton-grass or cotton-grass-sphagnum peat (Fig. 2, type I).

Most raised bogs contain only of a top layer of Sphagnum fuscum peat of 0.8 to $1.2 \mathrm{~m}$ thickness which developed on fens of sedges and herbaceous, rarely brown moss peat of 1.0 to $2.5 \mathrm{~m}$ thickness with a thin transitional Scheuchzeria or Eriophorum peat layer between. The fen peat types developed on Equisetum fluviatile peat, and on gyttia of 0.6 to $0.8 \mathrm{~m}$ depth indicating a lake environment at the start of the peat formation (Fig. 2, type II b). Outside of lake depression the same peat deposits may develop on waterlogged mineral soils (Fig. 2, type II a).

Fens are represented by three main types of peatlands: (1) sedge-Scheuchzeria mires developing in close water-encroached depressions on sandy soils, (2) herb-Sphagnum floating mires receiving run-off water or nutrient poor ground water (pure fens), and (3) Sedges and aapa mires developing under (waterlogged but) more nutrient rich conditions.

Type 1 fens consist of Scheuchzeria mires growing in depressions of the sandy uplands. Even though this type occupies only small areas of the contemporary peatland, this type is well presented in peat deposits both as initial stages of peat accumulation on sandy soils (Fig 2, type III a, core 25), which can be quite long, or as short transitional phase between nutrient rich fen and rain-fed bog layers in mixed peat deposits (Fig. 2, type II a, b, cores 14, 15).

Type 2 fens consist of herb-Sphagnum mires, which are usually connected to upper parts of small river valleys or creeks. They occur also between raised bogs and nutrient rich sedgemoss fens. They are characterized by Menyanthes trifoliata, Carex ssp. and Sphagnum obtusum. These mire types represent relative short-lived stages in peat and land surface development. 
Most common fen types are homogenous sedge and sedge-moss open (tree less) fens or with sparsely distributed small birch trees fed both by run-off and lateral flow of ground water. Aapa mires are characterized by patterned surface with narrow peat ridges (strings) oriented perpendicular to the direction of surface water flow. Sedges, such as Carex lasiocarpa, C. diandra, C. chordorrhiza, and the herbaceous Menyanthes trifoliata form the herb layer. Usually some brown moss species (Drepanocladus spp., Calliergon spp, Helodium blandowii) and some Sphagnum species (Sphagnum warnstorfii, S. subsecundum) are present. This vegetation delivers a sedge, sedge-herb, or brown moss-sedge peat, which are very widespread as 1.2 to $2.2 \mathrm{~m}$ deep fen deposits (Fig. 2, type III b, c, cores 5, 11, 17). The peat profiles consist often of 10-20-cm Sphagnum warnstorfii peat on the top (Fig. 2, core 5). In the deepest parts of the peatland 0.6-0.8 m horsetail peat (and gyttya) was formed beneath the sedge-fen deposit (Fig. 2, type III b, core 11). In one case $0.4 \mathrm{~m}$ basal layer of wood peat was found under sedge-fen deposit (Fig. 2, core 17).

Forested swamps consist of the mire types fed by ground water. These mire types are characterized by tall well developed tree layer (about 8-18 m high). The mire types occur within the river valleys and on the periphery of the main peatland covering the young terraces of Yenisey River. The vegetation is characterized with mixed forest of Pinus sibirica, Picea obovata, Betula pubescens and tussock sedges of Carex cespitosa, C. juncella, C. appropinquata in association with numerous herbs, graminoids and moss species.

Within the wide meander of river valleys forested swamps develop (without trees as Carex herb fens. They are distinct by) on deep (5-6 m) peat deposits combined by 0.8-1.0 m wood peat on top of less decomposed herbaceous (fern, Menyanthes) and Carex peat (Fig. 2, type $\mathrm{IV}$, core 28). The forested mire occurred on the periphery of the main peatlands developed on the shallow 0.5-1 m very well decomposed dense wood and sedge-wood peat deposits (Fig. 2, type $\mathrm{V}$, core 19 ).

The profiles and the peat stratigraphy of the main peat types are described in Table S1.

Table S1: Present day peatland types in relation to peat profiles. The peat-type layers are listed from top to bottom. Bold letters indicate the peat layers of more than $1 \mathrm{~m}$ thickness; brackets indicate peat layers less than $30 \mathrm{~cm}$. Bold numbers mark cores presented in Fig. 2.

Peat profile types $\left(4^{\text {th }}\right.$ column) contain (I) Shallow Sphagnum peat deposits representing mainly modern peat formation of raised bogs on sandy soils. (II) Mixed peat deposits in centers of well-developed raised bogs composed of 1-2 m deep fen peat covered by $0.5-1 \mathrm{~m}$ sphagnum raised bog peat layer. (III) Sedge-fen type peat deposits of Carex associated with through-flow of mineral-rich water from run-off or ground water including the initial transformation to rain-fed Sphagnum bogs. (IV) Deep herbaceous \& herb-sedge peat deposits sometimes covered at the later stage by woody peat in old river valleys. (V) Shallow woody peat deposits developed by forested swamps on the loamy soils which are widespread on the young left terrace of Yenisey River. The description of peat profile types contains also information on horizon depth.

\begin{tabular}{|l|l|l|l|l|l|l|}
\hline $\begin{array}{c}\text { Peatland } \\
\text { type }\end{array}$ & $\begin{array}{c}\text { Area, } \\
\mathbf{k m}^{2}\end{array}$ & $\begin{array}{c}\text { Area } \\
\mathbf{\%}\end{array}$ & \multicolumn{1}{|c|}{ Peat profile type } & $\begin{array}{l}\text { Depth, } \\
\mathbf{m}\end{array}$ & $\begin{array}{l}\text { Stratigraphy of } \\
\text { Peat types }\end{array}$ & $\begin{array}{c}\text { Number } \\
\text { of peat } \\
\text { cores** }\end{array}$ \\
\hline $\begin{array}{l}\text { A. } \text { Raised } \\
\text { bogs \& } \\
\text { ridge- } \\
\text { hollow } \\
\text { complexes }\end{array}$ & 2256.6 & 81 & $\begin{array}{l}\text { I. Shallow sphagnum } \\
\text { deposit }\end{array}$ & $0.5-1.5$ & $\begin{array}{l}\text { Sphagnum; Sphagnum- } \\
\text { Eriophorum }\end{array}$ & $\begin{array}{l}1,3,3 \mathrm{a}, \\
\mathbf{2 3}\end{array}$ \\
\hline
\end{tabular}




\begin{tabular}{|c|c|c|c|c|c|c|}
\hline & & & b) on gyttia & & $\begin{array}{l}\text { Sphagnum-Scheuchzeria- } \\
\text { Hypnum-(gyttija); } \\
\text { Sphagnum-Scheuchzeria- } \\
\text { Carex-Hypnum-Equisetum- } \\
\text { gyttia; }\end{array}$ & $\begin{array}{l}6,10,12, \\
13, \mathbf{1 4} ;\end{array}$ \\
\hline $\begin{array}{l}\text { B. Fens \& } \\
\text { aapa mires }\end{array}$ & 368.4 & 13 & $\begin{array}{l}\text { III. Sedge-fen type } \\
\text { a) transitional stage to } \\
\text { rain-fed bog; } \\
\text { Fen stage } \\
\text { b) on mineral soils } \\
\text { c) on gyttia }\end{array}$ & $1.0-1.5$ & $\begin{array}{l}\text { Carex-Scheuchzeria; } \\
\text { Sphagnum-Scheuchzeria; } \\
\text { Sphagnum-Carex- } \\
\text { Menyanthes; } \\
\text { Scheuchzeria-Carex- } \\
\text { Menyanthes; Scheuchzeria- } \\
\text { Carex; } \\
\text { (Sphagnum)-Scheuchzeria } \\
\\
\text { (Sphagnum)-Carex; } \\
\text { Hypnum-Carex-Wood; } \\
\text { (Scheuchzeria)-Carex- } \\
\text { Mentyanthes; Carex; } \\
\text { (Hyppnum)-Scheuchzeria- } \\
\text { Carex. } \\
\text { Carex-Mentyanthes- } \\
\text { Equisetum-gyttja; }\end{array}$ & $\begin{array}{l}\mathbf{5 , 1 7}, 21, \\
22,26\end{array}$ \\
\hline $\begin{array}{l}\text { C. } \\
\text { Forested } \\
\text { swamps \& } \\
\text { wooded } \\
\text { fens }\end{array}$ & 182.4 & 7 & $\begin{array}{l}\text { IV. Deep herb- } \\
\text { sedge-woody } \\
\text { deposits } \\
\text { V. Shallow woody } \\
\text { deposits }\end{array}$ & $\begin{array}{l}5.0-5.8 \\
0.5-1.5\end{array}$ & $\begin{array}{l}\text { Wood-Carex- } \\
\text { Menyanthes; } \\
\text { Sedge-wood; Wood }\end{array}$ & $\begin{array}{l}28,29 \\
19,20\end{array}$ \\
\hline
\end{tabular}

\section{Supplement S2: Carbon concentrations}

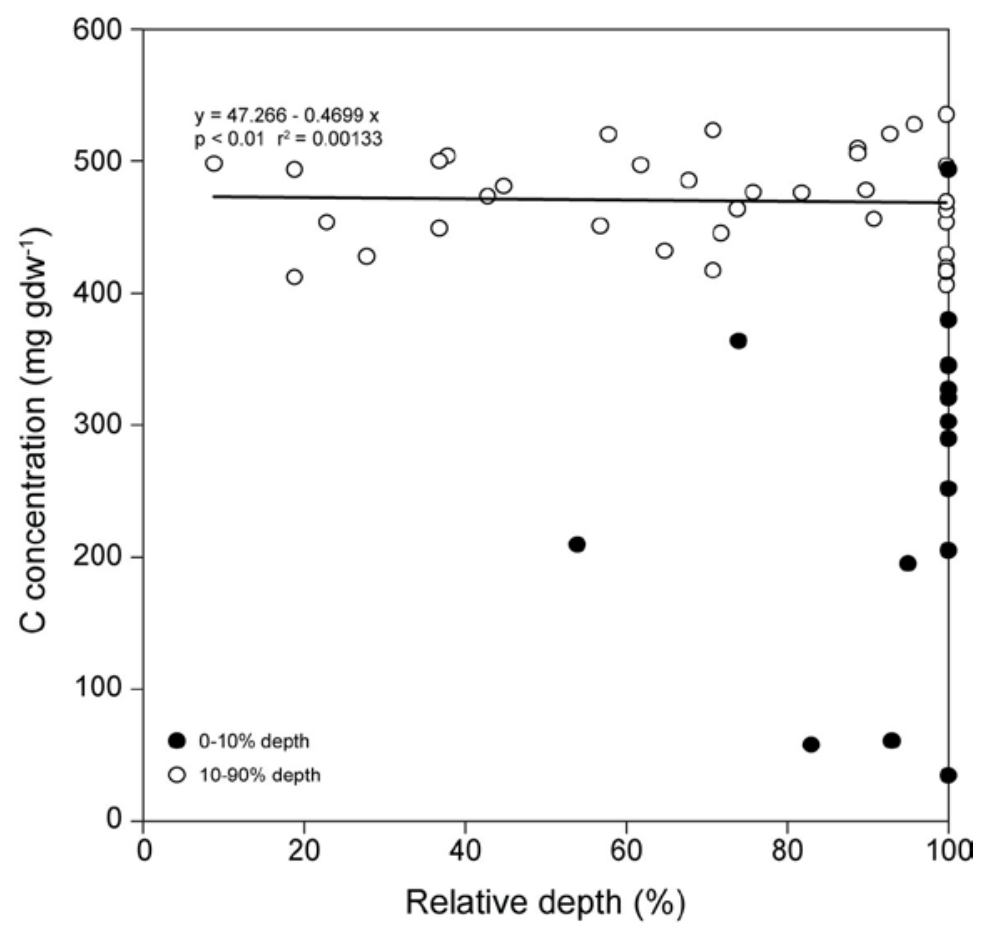

Fig. S2: Carbon concentrations as related to the relative bog depth. 100\% represents the total length of the core. Filled circles: 0 to $10 \%$ of total core length, un-filled circles: 10 to $90 \%$ of the total core length 


\section{Supplement S3}

Results of AMS $14 C$ analysis

Asterix * indicates data where a mix-up of samples occurred or age seqeunce was inverted

\begin{tabular}{|c|c|c|c|c|c|c|c|c|c|c|c|}
\hline \multirow[b]{2}{*}{ Core } & \multirow[b]{2}{*}{$\begin{array}{l}\text { Depth } \\
\text { (cm) }\end{array}$} & \multirow[b]{2}{*}{ Lab. code } & \multirow[b]{2}{*}{$\begin{array}{c}14 C \text { years } \\
\text { BP }\end{array}$} & \multicolumn{4}{|c|}{ POM } & \multicolumn{2}{|c|}{ DOC } & \multirow[b]{2}{*}{\begin{tabular}{|c|} 
Stage of \\
peatland type
\end{tabular}} & \multirow[b]{2}{*}{ Dated peat type } \\
\hline & & & & $\begin{array}{c}\text { Std } \\
\text { deviation }\end{array}$ & \begin{tabular}{|l} 
Calibrate \\
d 14C \\
age (yr \\
BP) POM
\end{tabular} & \begin{tabular}{|c|} 
Std \\
deviation \\
POM
\end{tabular} & Lab. code & \begin{tabular}{|c|} 
Calibrate \\
d 14C \\
age (yr \\
BP) DOC
\end{tabular} & $\begin{array}{c}\text { Std } \\
\text { deviation } \\
\text { DOC }\end{array}$ & & \\
\hline $1 \mathrm{a}$ & $102-103$ & 3613 & 4290 & 23 & 4852 & 10 & 3614 & 4036 & 49 & Fen (transitional & Carex \\
\hline 3 & $84-85$ & 3625 & 495 & 20 & 525 & 9 & 3626 & 510 & 9 & Raised bog & Sphanum \\
\hline $3 a$ & $76-77$ & 3619 & 2255 & 20 & 2260 & 76 & 3620 & 1485 & 62 & Raised bog & Eriophorum \\
\hline 4 & $114-115$ & 3609 & 3595 & 22 & 3910 & 48 & 3610 & 2610 & 107 & Fen & Menyanthes \\
\hline 4 & $119-120$ & 3611 & 3840 & 22 & 4225 & 65 & 3612 & 3765 & 60 & Fen & Menyanthes \\
\hline 5 & $49-50$ & 3593 & 400 & 17 & 485 & 15 & 3694 & 145 & 150 & Fen & Carex \\
\hline 5 & $99-100$ & 3607 & 3300 & 23 & 3525 & 40 & 3608 & 1980 & 53 & Fen & Carex \\
\hline 5 & $134-135$ & 3595 & 5655 & 25 & 6440 & 30 & 3596 & 5625 & 25 & Fen & Carex \\
\hline 6 & $280-281$ & 3621 & 9150 & 30 & 10310 & 65 & 3622 & 7895 & 40 & Shallow water & Equisetum \\
\hline 7 & $130-133$ & 3623 & 5060 & 25 & 5820 & 71 & 3624 & 3860 & 25 & Fen & Carex \\
\hline 8 & $195-196$ & 3617 & 8310 & 27 & 9345 & 60 & 3618 & 7220 & 40 & Fen & Menyanthes \\
\hline 9 & $200-201$ & 3615 & 8515 & 30 & 9520 & 17 & 3616 & 7885 & 45 & Fen & Menyanthes \\
\hline 10 & $295-296$ & 3508 & 10935 & 44 & 12885 & 25 & 3509 & 8885 & 105 & Lake & Gyttia \\
\hline 11 & $47-49$ & 3556 & 540 & 29 & 535 & 13 & 3557 & 620 & 50 & Fen & Carex \\
\hline 11 & $97-98$ & 3562 & 3287 & 22 & 3515 & 40 & 3563 & 2415 & 50 & Fen & Menyanthes \\
\hline 11 & $147-148$ & 3560 & 7032 & 29 & 7885 & 44 & 3561 & $7050^{\star}$ & 102 & Fen & Carex \\
\hline 11 & $197-198$ & 3554 & 8395 & 32 & 9445 & 35 & 3555 & 6085 & 90 & Fen & Menyanthes \\
\hline 11 & $247-248$ & 3558 & 10615 & 33 & 12715 & 55 & 3559 & $5965^{*}$ & 27 & Shallow water & Equisetum \\
\hline 11 & $277-278$ & 3564 & 11430 & 33 & 13285 & 37 & 3565 & 6710 & 34 & Lake & Gyttia \\
\hline 12 & $227-228$ & 3506 & 9183 & 45 & 10325 & 75 & 3507 & 8280 & 70 & Shallow water & Equisetum \\
\hline 13 & $40-50$ & 3575 & 250 & 18 & 295 & 10 & 3576 & 1950 & 0 & Raised bog & Sphanum \\
\hline 13 & $97-98$ & 3569 & 485 & 17 & 520 & 10 & 3570 & 1950 & 0 & Raised bog & Sphanum \\
\hline 13 & $147-148$ & 3577 & 2723 & 22 & 2815 & 30 & 3578 & 2610 & 115 & Fen & Scheuchzeria \\
\hline 13 & $197-198$ & 3587 & 6113 & 27 & 7045 & 102 & 3588 & 5125 & 150 & Fen & Carex \\
\hline 13 & $247-248$ & 3567 & 7455 & 25 & 8275 & 62 & 3568 & 4740 & 87 & Fen & Hypnum \\
\hline 13 & $297-298$ & 3571 & 8103 & 25 & 9040 & 40 & 3572 & 3767 & 118 & Fen & Hypnum \\
\hline 13 & $347-347$ & 3573 & 8568 & 30 & 9538 & 10 & 3574 & 8390 & 24 & Fen & Hypnum \\
\hline 14 & $398-399$ & $3110^{\star \star}$ & 12953 & 52 & 15286 & 140 & $3046^{*}$ & 11280 & 35 & Lake & Gyttia \\
\hline 15 & $49-50$ & 3585 & 290 & 20 & 363 & 62 & 3586 & 705 & 22 & Raised bog & Sphanum \\
\hline 15 & $99-100$ & 3579 & 640 & 20 & 610 & & 3580 & 1338 & & Raised bog & Sphanum \\
\hline 15 & $145-146$ & 3583 & 865 & 23 & 2230 & 75 & 3584 & 1360 & 21 & Fen & Menyanthes \\
\hline 15 & $166-167^{*}$ & 3516 & 2196 & 23 & $765^{\star}$ & 25 & 3517 & $855^{\star}$ & 55 & Fen & Menyanthes \\
\hline 15 & $199-200$ & 3581 & 6865 & 30 & 7695 & 30 & 3582 & 5380 & 70 & Fen & Menyanthes \\
\hline 15 & $224-225$ & 3589 & 8385 & 30 & 9403 & 70 & 3590 & 7065 & 92 & Forested swam & Carex-woody \\
\hline 17 & $47-48$ & 3601 & 160 & 18 & 142 & 133 & 3602 & 392 & 73 & Fen & Hypnum \\
\hline 17 & $97-98$ & 3597 & 1263 & 20 & 1218 & 39 & 3598 & 1950 & 0 & Fen & Carex \\
\hline 17 & $147-148$ & 3591 & 4155 & 22 & 4725 & 95 & 3592 & 2264 & 83 & Fen & Carex \\
\hline 17 & $197-198$ & 3599 & 7080 & 27 & 7912 & 41 & 3600 & 4695 & 115 & Fen & Carex \\
\hline 17 & $247-248$ & 3603 & 8830 & 29 & 9947 & 170 & 3604 & 6266 & 46 & Forested swam & Woody \\
\hline 17 & $262-263$ & 3605 & 8935 & 30 & 10070 & 120 & 3606 & 6264 & 46 & Forested swam & Woody \\
\hline 18 & $194-195$ & 3550 & 7910 & 30 & 8705 & 70 & 3551 & 6295 & 13 & Fen & Carex \\
\hline 19 & $88-89$ & 3366 & 3182 & 19 & 3412 & 31 & 3367 & 3595 & 31 & Forested swamf & Woody \\
\hline 20 & $39-40$ & 3552 & 1263 & 20 & 1218 & 39 & 3553 & 950 & 23 & Forested swamp & Woody \\
\hline 21 & $146-147$ & 3356 & 7900 & 24 & 8693 & 58 & 3358 & 8118 & 64 & Fen & Menyanthes \\
\hline 22 & $40-50$ & 3512 & 1245 & 25 & 1205 & 55 & 3513 & 1114 & 54 & Fen & Carex \\
\hline 22 & $99-100$ & 3514 & 3990 & 30 & 4470 & 45 & 3515 & 4355 & 60 & Fen & Carex \\
\hline 22 & $115-116$ & 3510 & 5655 & 35 & 6440 & 35 & 3511 & 5540 & 55 & Fen & Carex \\
\hline 23 & $99-100$ & 3362 & 3300 & 20 & 3521 & 38 & 3363 & 2615 & 100 & Pine bog & Eriophorum-woody \\
\hline 25 & $159-160$ & 3364 & 5990 & 20 & 6835 & 46 & 3365 & 5530 & 55 & Fen (transitional & Scheuchzeria \\
\hline 26 & $49-50$ & 3371 & 855 & 16 & 755 & 18 & 3372 & 1950 & 0 & Fen (transitional & Scheuchzeria \\
\hline 26 & $99-100$ & 3368 & 3570 & 19 & 3866 & 24 & 3369 & 3415 & 26 & Fen & Carex \\
\hline 26 & $149-150$ & 3504 & 6115 & 33 & 7046 & 107 & 3505 & 5380 & 68 & Fen & Menyanthes \\
\hline 26 & $199-200$ & 3370 & 7975 & 33 & 8879 & 101 & & & & Fen & Carex \\
\hline 26 & $220-221$ & 3373 & 8110 & 25 & 9044 & 36 & 3374 & 7345 & 70 & Fen & Carex \\
\hline 27 & $145-146$ & 3360 & 7620 & 25 & 8410 & 15 & 3361 & 7551 & 31 & Fen & Menyanthes-Carex \\
\hline 28 & $562-563$ & 3518 & 10180 & 40 & 11876 & 104 & 3519 & 9513 & 22 & Fen & Menyanthes \\
\hline 28 & $587-588$ & 3520 & 10450 & 44 & 12421 & 181 & 3521 & 9177 & 82 & Fen & Menyanthes \\
\hline 29 & $47-48$ & 3546 & 2185 & 25 & 2226 & 78 & 3547 & 1950 & 0 & Fen & Menyanthes-Carex \\
\hline 29 & $97-98$ & 3542 & 3365 & 25 & 3605 & 35 & 3543 & 2260 & 78 & Fen & Menyanthes-Carex \\
\hline 29 & $147-148^{*}$ & 3534 & 7360 & 66 & $8175^{\star}$ & 125 & 3535 & 2798 & 44 & Fen & Menyanthes-Carex \\
\hline 29 & $197-198$ & 3526 & 6080 & 34 & 6941 & 47 & 3527 & 5701 & 40 & Fen & Menyanthes-Carex \\
\hline 29 & $247-248$ & 3528 & 7162 & 37 & 7985 & 25 & 3529 & 5161 & 118 & Fen & Menyanthes-Carex \\
\hline 29 & $297-298$ & 3548 & 8085 & 30 & 9033 & 38 & 3549 & 5567 & 76 & Fen & Menyanthes-Carex \\
\hline 29 & $347-348$ & 3544 & 8815 & 35 & 9925 & 185 & 3545 & 12545 & 140 & Fen & Menyanthes-Carex \\
\hline 29 & $397-398$ & 3524 & 9345 & 40 & 10580 & 70 & 3525 & 7380 & 50 & Fen & Menyanthes-Carex \\
\hline 29 & $447-448$ & 3532 & 9915 & 40 & 11296 & 48 & 3533 & 5675 & 50 & Fen & Menyanthes-Carex \\
\hline 29 & $497-498$ & 3522 & 10230 & 40 & 11950 & 120 & 3523 & 8880 & 105 & Fen & Menyanthes-Carex \\
\hline 29 & $539-540^{*}$ & 3530 & 6900 & 35 & 7720 & 40 & 3531 & 6267 & 40 & Fen & Menyanthes-Carex \\
\hline
\end{tabular}

